\title{
Damping Low Frequency Oscillations in Power System using Quadratic Gaussian Technique based
}

\section{Control System Design}

\author{
Ibraheem K. Ibraheem, Ph. D \\ Department of Electrical Engineering \\ College of Engineering \\ Baghdad University
}

\begin{abstract}
The aim of this paper is to examine the effect of inclusion of a linear quadratic Gaussian controller instead of the conventional AVR with the existence of a PSS for improving the dynamic stability of power system. The present work introduces a computational methodology that adopted a Linear Quadratic Gaussian (LQG) controller to control the generator. In this method the models of both the synchronous generator and the PSS were assumed to be Linear, depending on this method the controller power consumption was minimized depending on some performance index, which is assumed to be Quadratic. The Two Degree of Freedom (2DOF) structure was adopted, in which two controllers are used, the first one is the LQG controller and the second one is the integral controller. The proposed controller has been checked and investigated with simulations run under Matlab environment on single machine infinite bus (SMIB) system and compared with the traditional design methods. From these results, it is clear that the LQG controller can enhances the steady state stability very clearly. The results also show that the use of the LQG, controller increases the damping torque that substitutes the need to the Power System Stabilizer (PSS).
\end{abstract}

\section{General Terms}

Power system stabilizer, synchronous generator, machine dynamics, excitation system.

\section{Keywords}

Linear quadratic Gaussian, integral control, alternating voltage regulator, single machine, steady state stability, twodegree-offreedom.

\section{INTRODUCTION}

The voltage regulator is the intelligence of the system and controls the output of the exciter so that the generated voltage and reactive power change in the desired way. In earlier systems the voltage regulator was entirely manual. Thus the operator observed the terminal voltage and adjusted the field rheostat (the voltage regulator) until the desired output conditions were observed. In most modern systems the voltage regulator is a controller that senses the generator output voltage (and sometimes the current) then initiates corrective action by changing the exciter control in the desired direction [1]. Rotating electrical machines play a very important role in the world's industrial life. In petrochemical and power utilities, the failure of critical rotating machines, such as motors or generators cost a lot of money. This is due to the loss of energy production, high emergency maintenance cost and lost revenues [2]. During the 1950s and into the 1960s, many power generating plants were equipped with continuously acting automatic voltage regulators. As the number of power plants with automatic voltage regulators grew, it became apparent that the high performance of these voltage regulators had a destabilizing effect on the power system. Power oscillations of small magnitude and low frequency often persisted for long periods of time. In some cases, this presented a limitation on the amount of power able to be transmitted within the system. Power system stabilizers were developed to aid in damping of these power oscillations by modulating the excitation supplied to the synchronous machine [3]. The prime mover plays a vital role in contributing to the stability of the whole system. Optimum transient responses of a closed loop control system to an external disturbance depends not just on the transfer function of the excitation controller, generator and sensors but also the speed/load controller as well [4]. Many researchers spot the light on the design of the AVR PSS systems, [5] Presents an on-line optimal approach for dynamic stability assessment of single-machine infinite-bus system. The approach is based on estimating the synchronizing and damping torque Coefficients of the synchronous machine. While [6] Shows the effect of changing the roots of the characteristics equation to a desired location in the complex plane, also in this research it is shown how this technique can improve the stability of a synchronous generator connected to infinite bus, authors in [7] presented a novel neuro controller for a synchronous generator based feedback signals from voltage, speed, active and reactive power. This controller is consists of two independent controllers' i.e. transient controller and voltage regulator. Examining the effect of inclusion of a power system stabilizer (PSS) in improving the dynamic stability of different power systems has been studied in [8]. A nonlinear dynamic model of PSS is applied here to study two electrical network configurations in order to show the influence of PSS controller to damp unstable inter-area electromechanical modes. In [9] the author presented a new structure for the AVR of the power system exciter is proposed and designed using digital-based LQR. Robust control is a very active control design tool especially when the system is MIMO and subjected to uncertainty and disturbance, standard H-infinity, $\mu$-synthesis, and recently LMI are all robust control techniques $[10,11,12]$. Many successful and powerful optimization methods and algorithms have been employed in formulating and solving the problem of AVR and PSS design. These optimization methodologies and techniques are widely diverse and have been the subject of ongoing enhancements over the years like swarm optimization techniques [13, 14, 15]. This paper is organized as follows: section 2 presents the mathematical modeling of the complete power system excitation module together with the machine dynamics. 
Controller design based on LQG technique is illustrated in section 3 , while the results are discussed in section 4 , the conclusion is given in section 5 .

\section{POWER SYSTEM MODELLING}

For any electric power system dynamic study, a proper mathematical model must be chosen. There are only a limited number of system components important to the dynamic study: the synchronous generator, the governor, PSS, and the excitation system. Fig.1 is representing the system structure including the PSS unit [16].

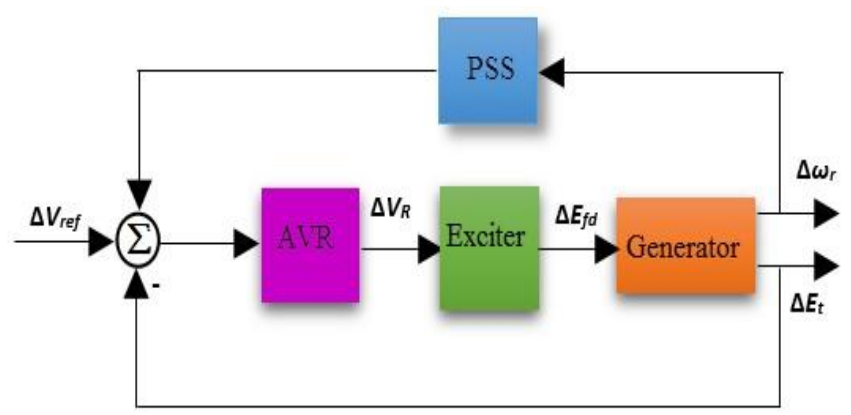

Fig 1: Block diagram of the power system model.

The performance of the excitation control system depends on the characteristics of excitation system, the generator and the power system. The basic function of the power system stabilizer (PSS) is to add damping to the generator rotor oscillations by controlling its excitation using auxiliary stabilizing signal(s). To provide damping, the stabilizer must produce a component of electrical torque in phase with the rotor speed deviations. Since the purpose of a PSS is to introduce a damping torque component, a logical signal to use for controlling generator excitation is the speed deviation. A simplified model describing the system dynamics used in this study is given by the following state space equations $[1,4$, $16]$.

$$
\begin{aligned}
& A=\left[\begin{array}{cccccc}
\frac{-K_{D}}{2 H} & \frac{-K_{1}}{2 H} & \frac{-K_{2}}{2 H} & 0 & 0 & 0 \\
\omega_{0} & 0 & 0 & 0 & 0 & 0 \\
0 & \frac{-K_{3} K_{4}}{T_{3}} & \frac{-1}{T_{3}} & \frac{K_{3}}{T_{3}} & 0 & 0 \\
0 & \frac{-K A K_{5}}{T_{E}} & \frac{-K A K_{6}}{T_{E}} & \frac{-K_{E}}{T_{E}} & 0 & 0 \\
\frac{-K_{D} K_{\text {STAB }}}{2 H} & \frac{-K_{1} K_{\text {STAB }}}{2 H} & \frac{-K_{2} K_{\text {STAB }}}{2 H} & 0 & \frac{-1}{T_{w}} & 0 \\
\frac{-T_{1} K_{D} K_{\text {STAB }}}{2 H T_{2}} & \frac{-T_{1} K_{1} K_{\text {STAB }}}{2 H T_{2}} & \frac{-T_{1} K_{2} K_{\text {STAB }}}{2 H T_{2}} & 0 & \frac{1}{T_{2}}-\frac{T_{1}}{T_{2} T w} & \frac{-1}{T_{2}}
\end{array}\right] \\
& B=\left[\begin{array}{llllll}
0 & 0 & 0 & \frac{K A}{T_{E}} & 0 & 0
\end{array}\right]^{T} \\
& C=\left[\begin{array}{llllll}
\mathrm{O} & K_{5} & K_{6} & \mathrm{O} & \mathrm{O} & \mathrm{O}
\end{array}\right] \\
& D=[\mathrm{O}] \\
& \text { and the state vector } x \text { of fig. } 2 \text { is defined as } \\
& x=\left[\begin{array}{llllll}
\Delta \omega_{r} & \Delta \delta & \Delta E^{\prime} & \Delta E_{f d} & \Delta v_{2} & \Delta v_{s}
\end{array}\right]^{T}
\end{aligned}
$$

where $\Delta$ refers to incremental change, and $\Delta \omega_{r}$ is the speed deviation, $\Delta \delta$ incremental change in rotor angle, $\Delta E^{\prime}$ incremental change in quadrature voltage, $\Delta E_{f d}$ incremental change in exciter voltage, $\Delta v_{2}$ incremental

\begin{tabular}{|c|c|c|}
\hline \multicolumn{3}{|c|}{ GENERATOR } \\
\hline$K D$ & Damping factor & 0 \\
\hline K1 & $\begin{array}{l}\text { Synchronous machine } \\
\text { factor }\end{array}$ & 0.7643 p.u. \\
\hline$K 2$ & $=$ & 0.8649 p.u. \\
\hline$K 3$ & $=$ & 0.323 p.u. \\
\hline$K 4$ & $=$ & 1.4187 p.u. \\
\hline$K 5$ & $=$ & -0.1463 p.u. \\
\hline K6 & $=$ & 0.4168 p.u. \\
\hline$T 3$ & $\begin{array}{c}\text { field circuit time } \\
\text { constant } \\
\end{array}$ & $2.365 \mathrm{sec}$ \\
\hline $2 H$ & $\begin{array}{c}\text { Mechanical starting } \\
\text { time }\end{array}$ & $3.5 \mathrm{sec}$ \\
\hline$f_{o}$ & frequency & $50 \mathrm{~Hz}$ \\
\hline \multicolumn{3}{|c|}{ EXCITER } \\
\hline$K A$ & $\begin{array}{c}\text { Conventional AVR } \\
\text { gain } \\
\end{array}$ & 30 \\
\hline$K E$ & Exciter gain & -0.02 \\
\hline$T E$ & Exciter time constant & $0.56 \mathrm{sec}$ \\
\hline \multicolumn{3}{|c|}{ POWER SYSTEM STABILIZER } \\
\hline KSTAB & PSS gsain & 9.5 \\
\hline$T_{W}$ & Washout time constant & $2.6 \mathrm{sec}$ \\
\hline$T 1$ & $\begin{array}{c}\text { Phase compensation } \\
\text { time constant }\end{array}$ & $0.4 \mathrm{sec}$ \\
\hline$T 2$ & $\begin{array}{l}\text { Phase compensation } \\
\text { time constant }\end{array}$ & $0.033 \mathrm{sec}$ \\
\hline
\end{tabular}
change in PSS washout voltage, $\Delta v_{s}$ incremental change in PSS output voltage. The detailed derivation of the above statespace matrices can be found in $[1,4,16]$. The values of the parameters of the above system are given in table 1 .

Table 1. Typical data of the power system model

The damping and synchronizing torque factors of tables 2, 3, and 4 are computed using the formulas[4]:

$$
\begin{aligned}
& \omega_{n}=\sqrt{K_{S} \frac{\omega_{o}}{2 H}} \mathrm{rad} / \mathrm{s} \\
& \zeta=\frac{1}{2} \frac{K_{D}}{2 H \omega_{n}}=\frac{1}{2} \frac{K_{D}}{\sqrt{K_{S} 2 H \omega_{o}}}
\end{aligned}
$$

\section{PROPOSED LQG CONTROL FOR STABILITY ENHANCEMENT}

The design of optimal feedback control systems for linear plants by using quadratic penalties on the state and control variables represents one of the most studied classes of problems in dynamic deterministic and stochastic optimal control theory. From a theoretical perspective, the so-called the Linear-Quadratic-Gaussian (LQG) problem (the $\mathrm{H}_{2}$ problem, as it is called these days) offer elegant methodologies amenable to numerical solution using generalpurpose CAD software. LQG control can be classified as an optimal control developed to achieve certain optimal performance. This could be well defined and easily formulated as optimization problems [17]. The proposed LQG controller structure is given in fig. 2. It consists of a feedback link that cascaded two main blocks namely the LQR $(K)$ and the Kalman Filter $\left(K_{e}\right)$. The reason for including the $K_{e}$ is to 
substitute for the noise, accurate plant models were frequently not available and the assumption of white noise disturbances (which is assumed Gaussian-distributed) has been taken to be corrupting the process. So the LQG problem and its solution can be separated into two distinct parts, LQR design and Kalman filter Design. Note that the state-space matrices in fig. 2 are that of the excitation-PSS system given in section 2 .

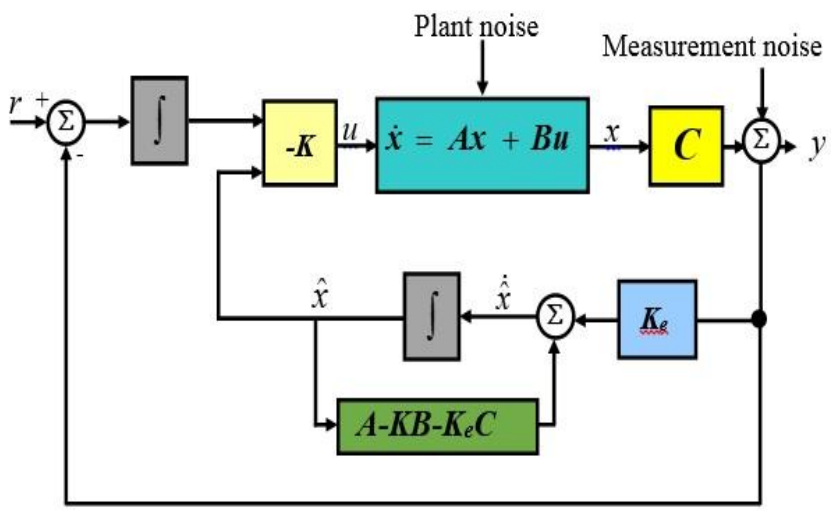

Fig 2: Proposed 2DOF LQG Control Structure for excitation system.

The design procedure for 2DOF LQG controller is summarized as follows [17]:

1. Design an LQR control law $\boldsymbol{u}=-\boldsymbol{K} x$ which solves the following problem,

$$
\begin{aligned}
& \qquad \dot{x}=A x+B u \\
& J_{(x, u, Q, R)}=\int_{0}^{\infty}\left(x^{T} Q x+R u^{2}\right) d t, Q \geq 0, R>0 \\
& \text { i.e., compute } \\
& A^{T} P+P A-P B R^{-1} B^{T} P+Q=0, P>0,
\end{aligned}
$$

2. Design a Kalman filter for the given plant, i.e.,

$$
\begin{gathered}
\dot{\hat{x}}=A \hat{x}+B u+K_{e}(y-\hat{y}), \\
\hat{y}=C \hat{x}, \text { where } \\
P_{e} A^{T}+A P_{e}+Q-P_{e} C^{T} R^{-1} C P_{e}=0, \\
P_{e}>0, \quad K_{e}=P_{e} C^{T} R^{-1}
\end{gathered}
$$

3. The LQG control law is given by $\boldsymbol{u}=-\boldsymbol{K} \hat{\boldsymbol{x}}$, i.e.

$$
\left\{\begin{array}{c}
\dot{\hat{x}}=A \hat{x}+B u+K_{e}(y-C \hat{x}) \\
u=-K \hat{x}
\end{array}\right.
$$

\section{PERFORMANCE EVALUATION OF} THE PROPOSED LQG CONTROLLER

To demonstrate the effectiveness and robustness of the proposed 2DOF LQG controller, A 555MW turbogenerator whose details are given in table 1 connected to infinite busbar system has been taken as a case study. Changing the value of KA from 1 to 91 , and for these changes evaluating the values of the KS, KD and the time domain specifications, as given in table 2. Fig. 3 shows the plotting of the output terminal voltage $E_{t}$ versus $\mathrm{t}$ for $\mathrm{KA}=1$ in the presence of PSS. The standard LQG design structure, which is a $1 \mathrm{DOF}$, does not give a controller with integral action. The mathematical model which represents the AVR designed depending on 1DOF LQG is the same as that of 2DOF LQG except that the integrator in the feed-forward path is removed. The values of $\mathrm{K}_{\mathrm{S}}$ and $\mathrm{K}_{\mathrm{D}}$ and the time domain specifications evaluated using 1DOF LQG controller are presented in table 3. The mathematical model which represents the AVR designed using 2DOF LQG and the generator has been simulated under MATLAB environment. The values of $K_{S}$ and $K_{D}$ and the time domain specifications evaluated using this structure are given in table 4 . Fig. 4 shows the plotting of the output terminal voltage $E_{t}$ versus $t$. What is obvious in the LQG technique (both $1 \mathrm{DOF}$ and $2 \mathrm{DOF}$ ) is the large value of the damping torque, and the time domain specification (control performance) is enhanced in the 2DOF LQG controller over that of 1DOF LQG one due to the effect of integral action. Table 5 shows a comparison between the proposed and conventional methods for calculating damping factor.

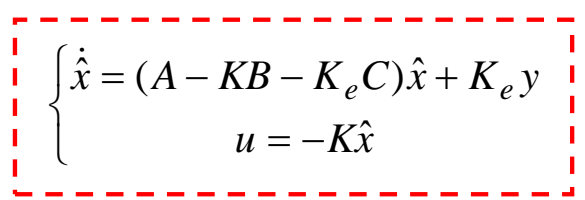




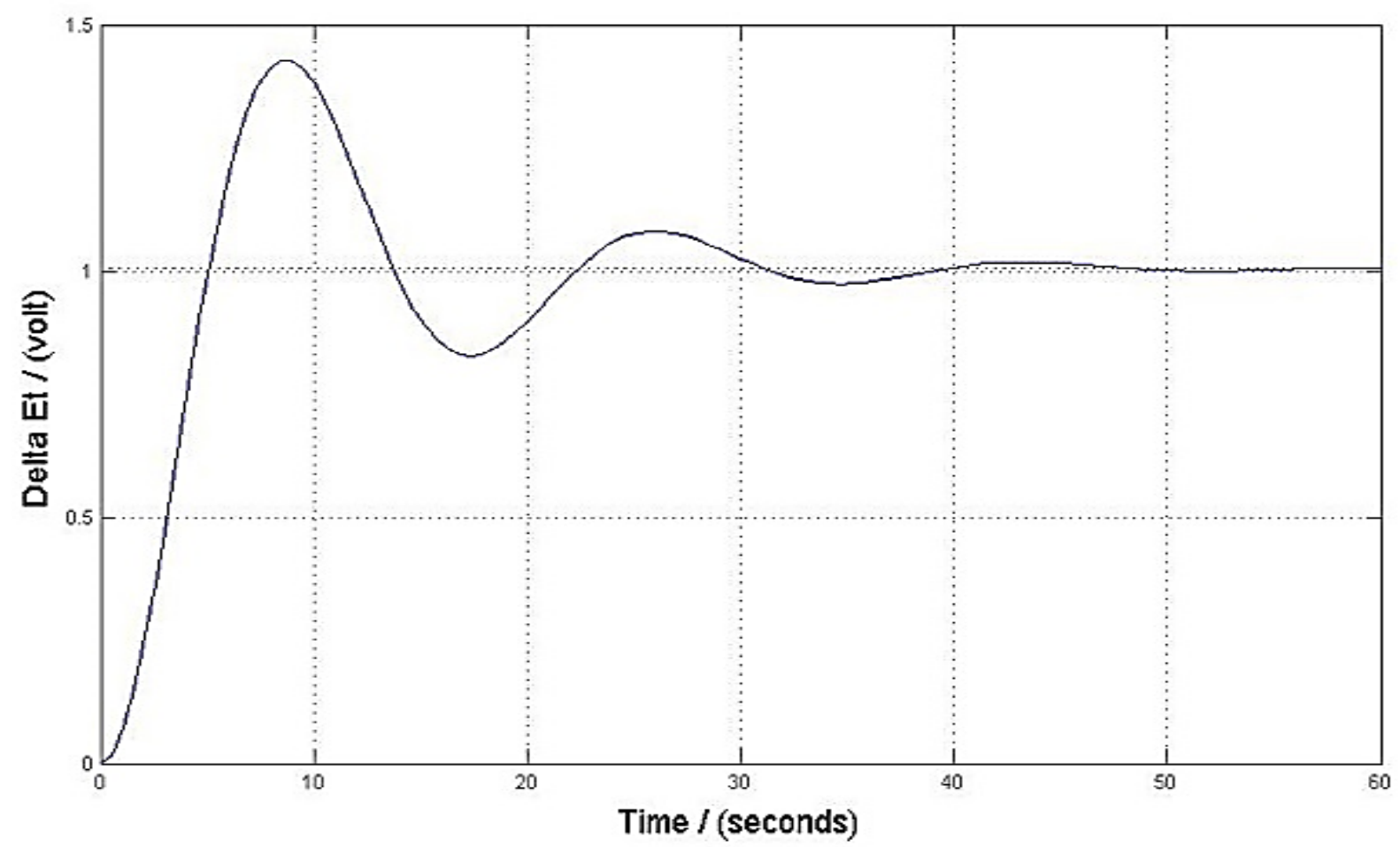

Fig 3: Terminal voltage of the generator with $\mathrm{KA}=1$ for AVR+PSS.

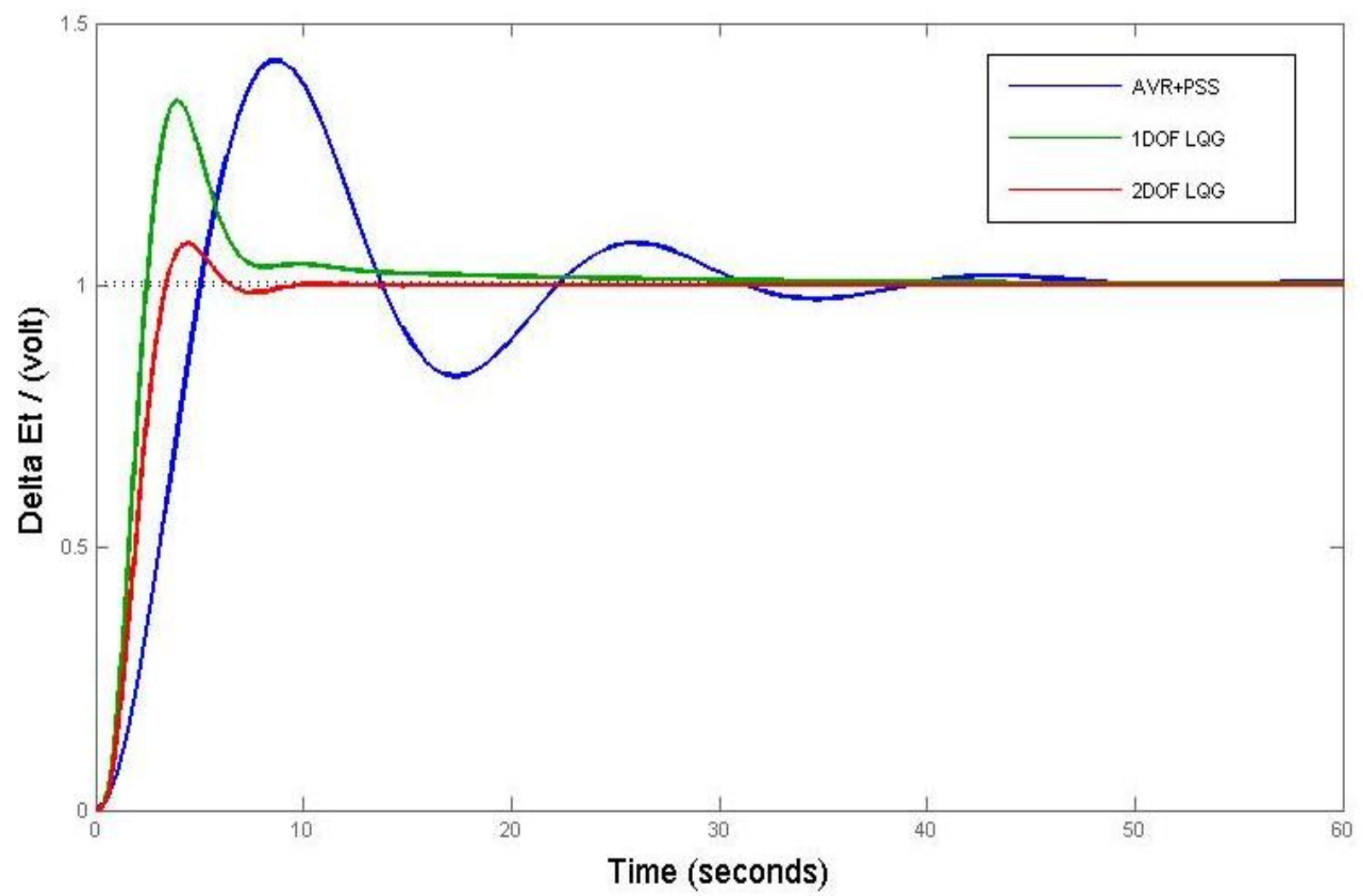

Fig 4: Terminal voltage of the generator using proposed LQG based AVR deign. 
Table 2. Conventional AVR + PSS results.

\begin{tabular}{|c|c|c|c|c|c|c|c|c|c|c|c||}
\hline $\mathbf{K A}$ & $\boldsymbol{\omega}_{\mathbf{n}}$ & $\boldsymbol{\omega}_{\mathbf{d}}$ & $\boldsymbol{\zeta}$ & $\mathbf{t}_{\mathbf{s}}$ & $\mathbf{K}_{\mathbf{s}}$ & $\begin{array}{c}\mathbf{K}_{\text {stotal= }} \\
\mathbf{K} \mathbf{1 + \mathbf { K } _ { \mathbf { s } }}\end{array}$ & $\mathbf{K}_{\mathbf{D}}$ & $\mathbf{t}_{\mathbf{r}}$ & $\begin{array}{c}\text { Peak } \\
\mathbf{A m p l}\end{array}$ & $\mathbf{t}_{\mathbf{p}}$ & $\boldsymbol{\%}_{\mathbf{p}}$ \\
\hline $\mathbf{1}$ & 0.37 & 0.36 & 0.2650 & 37.2 & 0.002 & 0.7668 & $\underline{\mathbf{1 . 3 9 5}}$ & 3.4 & 1.43 & 8.64 & 42.2 \\
\hline $\mathbf{1 0}$ & 1.2 & 1.2 & 0.0819 & 39.7 & 0.026 & 0.7910 & $\underline{\mathbf{1 . 3 7 5 9}}$ & 0.866 & 1.78 & 2.58 & 77.5 \\
\hline $\mathbf{2 0}$ & 1.71 & 1.71 & 0.0563 & 40.7 & 0.054 & 0.8185 & $\underline{\mathbf{1 . 3 4 7 8}}$ & 0.585 & 1.86 & 1.92 & 85.6 \\
\hline $\mathbf{3 0}$ & 2.11 & 2.11 & 0.0445 & 41.9 & 0.082 & 0.8469 & $\underline{\mathbf{1 . 3 1 4 5}}$ & 0.484 & 1.89 & 1.47 & 89.4 \\
\hline $\mathbf{4 0}$ & 2.46 & 2.45 & 0.0371 & 43.6 & 0.112 & 0.8766 & $\underline{\mathbf{1 . 2 7 7 7}}$ & 0.427 & 2 & 1.27 & 100 \\
\hline $\mathbf{5 0}$ & 2.77 & 2.77 & 0.0316 & 44.3 & 0.142 & 0.9067 & $\underline{\mathbf{1 . 2 2 5 4}}$ & 0.389 & 2.1 & 1.12 & 110 \\
\hline $\mathbf{6 0}$ & 3.08 & 3.07 & 0.0271 & 46.1 & 0.176 & 0.9404 & $\underline{\mathbf{1 . 1 6 8 6}}$ & 0.361 & 2.18 & 1.08 & 118 \\
\hline $\mathbf{7 0}$ & 3.37 & 3.37 & 0.0230 & 50.3 & 0.210 & 0.9751 & $\underline{\mathbf{1 . 0 8 5 1}}$ & 0.337 & 2.24 & 0.987 & 124 \\
\hline $\mathbf{8 0}$ & 3.67 & 3.67 & 0.0186 & 55.7 & 0.250 & 1.0143 & $\underline{\mathbf{0 . 9 5 5 7}}$ & 0.319 & 2.29 & 0.981 & 129 \\
\hline $\mathbf{9 0}$ & 4.00 & 4.00 & 0.0127 & 73.8 & 0.297 & 1.0613 & $\underline{\mathbf{0 . 7 1 1 2}}$ & 0.303 & 2.3 & 0.97 & 130 \\
\hline $\mathbf{9 1}$ & 4.04 & 4.04 & 0.0119 & 81 & 0.303 & 1.0673 & $\underline{\mathbf{0 . 6 7 3 1}}$ & 0.301 & 2.31 & 0.957 & 132 \\
\hline
\end{tabular}

Table 3. AVR design using 1DOF LQG.

\begin{tabular}{|c|c|c|c|c|c|c|c|c|c|c||}
\hline$\omega_{\mathbf{n}}$ & $\boldsymbol{\omega}_{\mathbf{d}}$ & $\boldsymbol{\zeta}$ & ts & $\mathbf{K s}$ & $\begin{array}{c}\text { Kstotal } \\
=\mathbf{K} 1+\mathbf{K} \mathbf{K}\end{array}$ & $\mathbf{K D}$ & $\mathbf{t r}$ & $\begin{array}{c}\text { Peak } \\
\text { Amp. }\end{array}$ & tp & \% MP \\
\hline 1.12 & 0.968 & 0.507 & 17.2 & 0.0233 & 0.78759 & $\underline{\mathbf{7 . 9 4 9 8}}$ & 1.51 & 1.35 & 4 & 35.1 \\
\hline
\end{tabular}

Table 4. AVR using proposed 2DOF LQG.

\begin{tabular}{|c|c|c|c|c|c|c|c|c|c|c||}
\hline$\omega_{\mathbf{n}}$ & $\boldsymbol{\omega}_{\mathbf{d}}$ & $\zeta$ & ts & Ks & $\begin{array}{c}\text { Kstotal } \\
=\mathbf{K 1 + K s}\end{array}$ & KD & tr & $\begin{array}{c}\text { Peak } \\
\text { Amp. }\end{array}$ & tp & \%MP \\
\hline 1.12 & 0.968 & 0.507 & 5.92 & 0.0233 & 0.78759 & $\underline{\mathbf{7 . 9 4 9 8}}$ & 2.04 & 1.08 & 4.46 & 8.04 \\
\hline
\end{tabular}

Table 5. Comparison of daming factor values calculated using different methods

\begin{tabular}{|c|c||}
\hline \hline Techniques & $\begin{array}{c}\text { Damping torque coefficient in } \\
\text { p.u. torque/p.u. speed deviation }\end{array}$ \\
\hline Conventional AVR & Max=1.395 to Min=0.0333 \\
\hline Conventional AVR + PSS & Max=1.395 to Min=0.6731 \\
\hline 1DOF LQG technique & 7.9498 \\
\hline 2DOF LQG technique & 7.9498 \\
\hline \hline
\end{tabular}




\section{CONCLUSION}

In this work a study of a simulated mathematical model for the excitation system together with the synchronous machine has been introduced. A controller based on a two degree of freedom Linear Quadratic Gaussian (2DOF LQG) has been proposed and designed to control generator terminal voltage. This technique, which depends on the LQG, gives best damping torque in addition to improving time domain performance of the system in comparison with the traditional techniques. The increased value of the damping torque improved the stability of the whole system against plant and measurement noise. The proposed 2DOF LQG controller showed excellent results as compared to the conventional AVR+PSS and 1DOF LQG and can be considered as promising tool for the control design of the excitation system not just on SMIB but to multi-machine power systems too.

\section{REFERENCES}

[1] P. M. Anderson, A. A. Fouad. 2002. Power System Control and Stability. $2^{\text {nd }}$ edition. Wiley-IEEE press.

[2] H. G. Sedding, B.A Lloyd, G.C Stone, J.M Braun, J.C White. 1989. Development of novel instrumentation and expert system concepts in turbine generator condition monitoring. Fourth International Conference on Electrical Machines and Drives.

[3] M. J. Basler, R.C Schaefer. 2007. Understanding Power System Stability. Pulp and Paper Industry Technical Conference, conference Record of Annual.

[4] P. Kundor. 1994. Power system stability and control, McGraw-Hill, Inc.

[5] E. A. Feilat, N Younan. 1999. On-line adaptive assessment of the synchronizing and damping torque coefficients using Kalman filtering. IEEE Proceedings Southeastcon ' 99.

[6] Zwe-Lee Gaing. 2004. A Particle Swarm Optimization Approach for Optimum Design of PID Controller in AVR System, IEEE Trans. on Energy Conversion, Vol. 19, No. 2

[7] R. Mohammadi-Milasi, M. J. Yazdanpanah, P. JabehdarMaralani. 2004. A novel adaptive gain-scheduling controller for synchronous generator. Proceedings of the 2004 IEEE International Conference on Control Applications, Vol. 1.
[8] Omer M. Awed-Badeeb. 2006. Damping of electromechanical modes Using power system stabilizers (pss)Case: electrical yemeni network. Journal of Electrical Engineering, VOL. 57, NO. 5, 291-295.

[9] I. K. Ibraheem. 2011. A Digital-Based Optimal AVR Design of Synchronous Generator Exciter using LQR Technique. ", Al-Khwarizmi Engineering Journal, ISSN 1818-1171, v. 7, no. 1

[10] M. Bouhamida, A. Mokhtari, M. A. Denai. 2005. Power System Stabilizer Design Based on Robust Control Techniques. ACSE Journal, Volume (5), Issue (3).

[11] Y. Peng, H. Nouri, $\quad$ Q. M. Zhu, L. Cheng. 2011. Robust Controller Design Survey for Damping Low Frequency Oscillations in Power Systems. Power and Energy Engineering Conference (APPEEC), 2011 AsiaPacific.

[12] [11] Y. Peng, Q. M. Zhu, H. Nouri. 2011. Robust $H_{2}$ Power System Stabilizer Design using LMI Techniques. Proceedings of International Conference on Modelling, Identification and Control, Shanghai, China, June 26-29.

[13] O. Abedinia, B. Wyns, A. Ghasemi. 2011. Robust Fuzzy PSS Design using ABC. 10th International Conference on Environment and Electrical Engineering (EEEIC), 811 May, pp. $1-4$.

[14] M. Marsaline Beno, N. Albert Singh, M. Ciba Therase, M. Mohamed Syed Ibrahim. 2011. Design of PSS for damping low frequency oscillations using Bacteria foraging tuned non-linear Neuro-Fuzzy Controller. IEEE GCC conference and exhibition, Dubai, Feb. 19-22.

[15] M. Eslami, H. Shareef, A. Mohamed. 2011. Power system stabilizer design based on optimization techniques. $4^{\text {th }}$ International Conference on Modeling, Simulation and Applied Optimization (ICMSAO), 19-21 April.

[16] H. Saadat. 1999. Power System Analysis, McGraw-Hill Inc.

[17] S. Skogested and I. Postlethwaite. 2005. Multivariable Feedback Control: Analysis and Design $2^{\text {nd }}$ edition,John Wiley \& Sons, Ltd 